\title{
Importância do Acinetobacter baumannii no ambiente hospitalar
}

\author{
Edson Wagner Silva Cangussu ${ }^{a^{*}}$ \\ ${ }^{a}$ Universidade Federal do Tocantins (UFT), Brasil \\ *Autor correspondente (edsonwcangussu@gmail.com)
}

\section{N F O}

\section{Keyworks}

health

infectious

mechanical ventilation

\begin{abstract}
A B S T R A C T
Importance of Acinetobacter baumannii in the hospital environment

Acinetobacter species are known as important opportunistic pathogens, especially in immunocompromised and hospitalized patients. Frequently the spread of $A$. baumannii occurs contact between patients, or through health professionals, directly or indirectly. A. baumannii has been the focus of attention of the scientific and medical community. In light of the foregoing, this paper aims to describe, through systematic literature review, about this species, its potegenicity and virulence, as well as to report on its prevalence and incidence. infectious agent in the hospital room. A bibliographic survey was carried out in Portuguese and English periodicals from 2000 to 2019 in the MEDLINE and LILACS databases. 34 bibliographic works were analyzed and inserted. Acinetobacter species have a range of virulence factors that enable survival and adaptation of microorganisms in the environment and resistance acquisition is accomplished by a variety of mechanisms. Immunocompromised and mechanically ventilated patients are commonly colonized and infected with this bacterial species. Based on the literature, it can be seen that $A$. baumannii has gained prominence and relevance as an infectious agent in the hospital environment. In particular, its role as an etiologic agent in ventilator-associated pneumonia shows how important strategies for prophylactic and preventive methods.
\end{abstract}

\section{R E S U M O}

As espécies de Acinetobacter são conhecidas como importantes patógenos oportunistas, principalmente em pacientes imunocomprometidos e hospitalizados. Frequentemente a disseminação de A. baumannii ocorre através do contato entre pacientes, ou através dos profissionais de saúde, direta ou indiretamente. $\mathrm{O}$ A. baumannii tem sido o cerne da atenção da comunidade científica e médica. Face o exposto o presente trabalho visa descrever, através de revisão literária sistemática, sobre essa espécie, sua patogenicidade e virulência, assim como relatar sobre a prevalência e incidência como agente infeccioso no ambiente hospitalar. Realizou-se um levantamento bibliográfico em periódicos das línguas portuguesa e inglesa, no perídio de 2000 a 2019, nas bases de dados MEDLINE e LILACS. Foram analisados e inseridos no trabalho 34 trabalhos. As espécies do gênero Acinetobacter apresentam uma gama de fatores de virulência que viabilizam a sobrevida e adaptação dos micro-organismos no ambiente e a aquisição de resistência é realizada por um número variado de mecanismos. Os pacientes imunocomprometidos e submetidos a ventilação mecânica são comumente colonizados e infectados por essa espécie bacteriana. Com base no levantamento bibliográfico pode-se aferir que o A. baumannii a cada dia tem ganhado destaque e relevância como agente infecioso no ambiente hospitalar. De forma especial sua participação como agente etiológico em pneumonia associada à ventilação mecânica mostra o quanto é importante estratégias visando métodos profiláticos e preventivos. 


\section{INTRODUÇÃO}

As infecções causadas por bactérias multirresistentes constituem um grave problema na saúde pública em todo o mundo. Essas infecções são responsáveis por aumentar consideravelmente a morbidade, a mortalidade e os custos com saúde (El Mekes et al., 2019).

Segundo a Agência Nacional de Vigilância Sanitária (ANISA, 2010) define como micro-organismos multirresistentes aqueles que apresentam resistência a diferentes classes de antimicrobianos testados em exames microbiológicos; e micro-organismos pan-resistentes aqueles que tem resistência comprovada in vitro a todos os antimicrobianos testados em exame microbiológico.

Estima-se que nos Estado Unidos mais de 70\% das bactérias isoladas no ambiente hospitalar apresentam resistência a pelo menos um antibiótico comumente utilizado no tratamento de infeções. A transmissão dos micro-organismos ocorre, comumente, pelo contato das mãos dos profissionais com os pacientes e pelo contato direto do paciente com material ou ambiente contaminado (Sales et al., 2014).

O gênero Acinetobacter pertence à família Moraxellacea, compreendendo cocobacilos Gram negativos que não apresentam motilidade, incapazes de fermentar a glicose e oxidase negativos. A espécie mais comum encontrada em amostras clínicas humanas é a. baumannii (Koneman et al., 2008). As espécies de Acinetobacter possuem elevado ecletismo metabólico e nutricional, adaptando-se facilmente a diferentes ambientes (Pereira, 2013). As espécies de Acinetobacter são facilmente encontradas na natureza, sendo a água e o solo o seu principal habitat. Espécies de Acinetobacter são facilmente e comumente isolados de uma gama de fontes, desde produtos lácticos, alimentos congelados, bem como em amostras de origem humana de indivíduos saudáveis tais como pele, conjuntiva, leite humano, garganta e uretra (Vergasa e Nieves, 2005).

Devido ao aparecimento dos primeiros surtos de infecções em hospital, no início dos anos 80, as infecções por A. baumannii têm sido uma questão clínica muito importante também no continente europeu (Duarte, 2011).

Uma razão para o crescente interesse atual no $A$. baumannii é sua perceptível capacidade de adquirir genes de resistência, levando ao surgimento de cepas multirresistentes. Além disso essa espécie exibe capacidade de sobreviver por longos períodos no ambiente hospitalar, corroborando com sua habilidade para surtos nosocomiais (Ciello e Araujo, 2016).
O A. baumannii tem sido o cerne da atenção da comunidade médica hospitalar. Sua continuidade e constância nos ambientes hospitalares, a relação que se instaura com o hospedeiro assim como a multirresistência tornou-o um micro-organismo temido e um grave problema para a saúde pública (Coelho, 2012)

Face o exposto o presente trabalho visa descrever, através de revisão literária sistemática, sobre essa espécie, sua a patogenicidade e virulência, assim como relatar sobre a prevalência e incidência como agente infeccioso no ambiente hospitalar.

\section{MATERIALES E MÉTODOS}

Realizou-se um levantamento bibliográfico em periódicos das línguas portuguesa e inglesa de 2000 a 2019 nas bases de dados: Bibliografia Médica (MEDLINE) e Literatura Latino-Americana e do Caribe em Ciências da Saúde (LILACS). Optou-se por trabalhos neste período visando uma perspectiva atual concernente ao tema, assim como um recorte temporal nas últimas duas décadas. Os critérios de inclusão foram: artigos de revisão e originais, assim como dissertações e teses. Foram analisados e inseridos no trabalho 34 trabalhos. Um livro foi acrescido também como fonte de fundamentação teórica devido sua relevância no ramo da microbiologia.

\section{RESULTADOS E DISCUSSÃO}

\section{Acinetobacter baumannii}

Espécies de Acinetobacter configuram importantes patógenos oportunistas. No contexto hospitalar, esses micro-organismos são associados a vários tipos de infecções, principalmente, em pacientes criticamente debilitados e imunocomprometidos. Estas infecções variam desde infecções da pele, tecidos moles, feridas e trato urinário a quadros de pneumonia, meningite e bacteremia. Infecções e surtos hospitalares estão sendo referidos, especialmente, à espécie A. baumannii e, em certo grau, as outras espécies, tais como: A. nosocomialis e A. pittii (Chagas, 2015).

A ocorrência de A. baumannii, que apresenta resistência a múltiplas drogas, tem aumentado de forma drástica nas duas últimas décadas, acompanhada da acentuada queda do desenvolvimento de antimicrobianos eficazes contra diversas espécies bacterianas (Verma e Tiwari, 2018). O A. baumannii não compartilha similaridade no quesito habitat com outras espécies do gênero Acinetobacter, havendo pouca evidência, que este seja residente no 
solo (Torres et al., 2010).

O cultivo in vitro das espécies de Acinetobacter, demonstra, em meios de cultura, colónias planas de coloração branco-acinzentada apresentando diâmetro entre de 1,5 a $3 \mathrm{~mm}$, em aerofilia à temperatura de $37^{\circ} \mathrm{C}$. A identificação das diferentes espécies é muito relevante sendo que existem sistemas e fluxogramas baseados em características bioquímicas, padrões de susceptibilidade aos antibióticos, sorotipagem, identificação de fagos e perfil proteico. A identificação das diferentes espécies é muito relevante sendo que existem sistemas e fluxogramas baseados em características bioquímicas, padrões de susceptibilidade aos antibióticos, sorotipagem, identificação de fagos e perfil proteico (Peleg et al. 2008; Koneman et al., 2008).

\section{Virulência}

As espécies de Acinetobacter apresentam uma gama de fatores de virulência que viabilizam a sobrevida e adaptação dos micro-organismos no ambiente. Exemplos de fatores virulência são: captação de ferro do meio ambiente, resistência à secagem, produção de uma cápsula polissacarídica, aderência a superfícies através da formação de biofilmes, e aderência às células epiteliais por meio de pili (Silva, 2009; Gusatti et al., 2009).

Os lipopolissacarídeos (LPS) relacionam-se na resistência ao complemento humano e agem em sinergismo com a cápsula exopolissacarídica. A cápsula polissacarídica é efetiva ao bloquear o acesso do complemento à parede celular bacteriana e previne a ativação. A formulação de exopolissacarídeo por bactéria patogênica é um fator de virulência e tem-se o conhecimento que os mesmos protegem a bactéria das defesas do hospedeiro. Estudos experimentais, utilizando cepas de Acinetobacter produtoras de exopolissacarídeo demonstraram maior patogenicidade nessas cepas do que as não produtoras, especialmente em infecções polimicrobianas com outras espécies de elevada virulência (Luiz, 2006).

A. baumannii sob condições de deficiência de ferro, é capaz de secretar moléculas envolvidas na captação de ferro, denominadas sideróforos, dentre essas o acinetobactin. A secreção de sideróforos é importante, uma vez que viabiliza o crescimento bacteriano e a expressão de fatores de virulência (Pereira, 2013; Zimbler et al., 2009).

Um importante fator de virulência dos biofilmes é a capacidade de adesão dos micro-organismos a superfícies inertes, especialmente, material médico invasivo como cateteres, próteses ou pacemakers (Heriques et al., 2013). Estudos tem demonstrado que a adesão das células de $A$. baumannii a super- fícies bióticas e abióticas ocorre por ativação de sistemas, tais como CsuA/BABCDE que estimula a produção de pili, proporcionando assim a aderência das células às superfícies abióticas (Pereira, 2013). A produção de biofilme culmina em aumentar sua virulência e diminuir sua suscetibilidade aos agentes antimicrobianos, isso favorece sua sobrevivência sob condições de crescimento desfavoráveis (Choi et al., 2008).

Segundo Zarrilli et al. (2004) as cepas de Acinetobacter apresentam características únicas dentre as bactérias Gram-negativas nosocomiais que favorecem sua persistência no ambiente hospitalar. Esse gênero apresenta alta resistência à dessecação, assim sendo persistem em ambientes secos e inanimados por longos períodos, podendo a mais de três semanas.

Moléculas de quorum sensing, como as lactonas $\mathrm{N}$-acil-homoserina (AHLs), possibilitam que algumas bactérias Gram-negativas respondam às mudanças ambientais através de comportamentos. Acinetobacter spp. também são produtoras de AHLs, a espécie A. baumannii formula uma AHL capaz de afetar sua motilidade e formação de biofilme (Dou et al., 2017). Estudos tem demonstrado que o fator quorum sensing é capaz de regular diversos processos metabólicos, incluindo a expressão de genes de virulência, bioluminescência, produção de toxinas, motilidade, diferenciação celular, esporulação, transferência plasmidial e formação de biofilmes (Pereira, 2013).

\section{Resistência}

Define-se como resistência natural, a resistência adquirida por mecanismos naturalmente presentes nos micro-organismos, que comumente conferindo resistência a uma classe de agentes antimicrobianos. Além do supracitado, as bactérias apresentam a habilidade de adquirir resistência, onde inicialmente populações suscetíveis de bactérias tornamse resistentes a um agente antimicrobiano por meio de seleção e mutações ou através da aquisição de informações de genes de outras bactérias que codificam a resistência (Sales et al., 2014).

A aquisição de resistência do A. baumannii é realizada por um número variado de mecanismos, incluindo a aquisição de elementos genéticos móveis (transposons, plasmídeos e integrons), assim como através de transformação natural. São observados mecanismos de resistência que envolvem a degradação enzimática, bombas de efluxo, deficiência de porinas e modificação do alvo (Ciello e Araujo, 2016).

O mecanismo primordial de resistência aos betalactamicos do A. baumanni consiste na produção de beta-lactamases. Assim como outros bacilos gram- 
negativo as beta-lactamases da classe $\mathrm{C}$, (cefalosporinases) codificadas cromossomicamente (AmpC) (Silva, 2009). A resistência às penicilinas, cefalosporinas e aztreonam é mediada especialmente por expressão genética de $\beta$-lactamases de espectro estendido (ESBL) e, comumente, motivador dos fenótipos de multi-resistência. As ESBLs das famílias TÊM, PER, VEB, CTX-M são muito frequentes nesse gênero (Gustti et al., 2009).

Além disso diversas beta-lactamases adquiridas pertencentes à classe $\mathrm{B}$, denominadas metalo-betalactamases (MBL), ou à classe $\mathrm{D}$, também conhecidas como oxacilinases, têm sido identificadas rotineiramente nestes micro-organismos (Figueiredo et al., 2009).

Vários mecanismos de resistência aos aminoglicosideos são observados, dentre eles, destaca-se a expressão de enzimas modificadoras como as acetiltransferases, fosfotransferases e nucleotidiltransferases. As bombas de efluxo e a metilação do rRNA também conferem resistência aos aminoglicosídeos (Peleg et al., 2008).

A resistência comumente descrita às fluoroquinolonas é mediado por mutações nas regiões determinantes da resistência às quinolonas (QRDRs) de gyrA que codifica a subunidade da DNA girase A e o parC que codifica a subunidade topoisomerase IV. Há também a resistência mediada por bombas de efluxo, como AdeABC e AdeM (Chopra \& Galande, 2011; Peleg et al., 2008).

Bombas de efluxo apresentam alta frequência em Acinetobacter spp., destacando-se o sistema de efluxo AdeABC, responsável pela resistência aos aminoglicosídeos, eritromicina, cloranfenicol, trimetoprim, fluoroquinolonas, cefotaxima e tetraciclina (Poole, 2004).

Mecanismos de resistência às tetraciclinas, em Acinetobacter consistem na produção de uma proteína protectora do ribossoma codificada pelo gene tet(M), bombas de efluxo e específicas das tetraciclinas tet(A) e tet(B) e também comuns a várias drogas. Há também relatos de resistência a outros antibióticos como à tigeciclina, ao trimetropim-sulfametoxazol e até mesmo às polimixinas (Gales et al. 2006; Perez et al., 2007).

\section{Epidemiologia}

Rodrigues et al. (2009) ao avaliarem 233 pacientes sob ventilação mecânica observou que 64 desenvolveram pneumonia associada à ventilação mecânica (PAVM) ao longo do seguimento do estudo, os micro-organismos isolados de maior incidência foram A. baumannii (28\%), P. aeruginosa (19\%) e S. aureus (20\%).

A pneumonia é uma importante consequência de infecção hospitalar, sendo frequente, em mais de
90\% dos casos, em pacientes submetidos à intubação endotraqueal e ventilação mecânica. Diversos estudos têm buscado caracterizar as pneumonias associadas a ventilação mecânica em termos de predisposições, prognóstico, microbiologia e custos (Rodrigues et al., 2009; Rello et al., 2002).

Comumente as pneumonias tardias se relacionam à própria ventilação mecânica, ou seja, são secundárias à colonização das vias aéreas superiores e à aspiração de secreções contaminadas. Em sua grande maioria são causadas por patógenos resistentes aos antimicrobianos como $P$. aeruginosa, $S$. maltophilia, espécies de Acinetobacter e Staphylococcus aureus resistentes à meticilina (MRSA) (Costa et al., 2016).

Sales et al. (2014) em um estudo transversal ao analisarem equipamentos/materiais e mobiliários com estreito contato com os pacientes e profissionais na UTI, observou que dentre as 49 amostras analisadas, $24,4 \%$ apresentaram presença de $A$. baumannii multirresistente. Os objetos que obtiveram positividade foram: respirador, bomba de infusão, estetoscópio, grades da cama e mesa de evolução clínica. Todas a cepas isoladas apresentaram resistência as cefalosporinas, carbamazepênico, quinolonas e nitrofuranos. Em contrapartida todas as cepas isoladas apresentavam sensibilidade a polimixina, glicilciclina e aminoglicosídeos.

Oliveira e Damasceno (2010) ao tentarem identificar na literatura a ocorrência de contaminação das superfícies inanimadas, relataram que em superfícies da cama os micro-organismos de grande incidência são Enterococcus, resistente à vancomicina (VRE), P. aeruginosa, C. difficile, A. baumannii e MRSA. O MRSA apresentou alta frequência em maçanetas, cadeiras, assentos sanitários e mesa, já a $P$. aeruginosa apresentou-se comumente em locais úmidos.

Uc-Cachón et al. (2019) em um estudo retrospectivo em isolados clínicos proveniente de setores hospitalares observaram que as estirpes de $\mathrm{A}$. baumannii exibiram altas taxas de resistência a cefalosporinas, ciprofloxacina, gentamicina e trimeto$\mathrm{prim} / \mathrm{sulfametoxazol}$.

Ciello e Araujo (2016) analisaram a prevalência de A. baumannii resistente a carbapenens no ambiente hospitalar no período de 2008 a 2014, observaram que das 420 amostras resistentes, os setores de maiores prevalências foram; Pronto Socorro $(26,4 \%)$ e CTI $(22,8 \%)$. Quanto ao sitio de infecção as amostras provenientes de secreções de ferida foram responsáveis por $25 \%$, seguidas pelas amostras de urina um percentual $21,4 \%$.

Esses achados corroboram com Davis et al. (2005) ao afirmarem que pacientes com queimaduras graves compõem grupo de alto risco para a co- 
lonização e posterior infecção pela espécie. Segundo os mesmos autores os pacientes queimados comumente desenvolvem infecções mais severas como bacteremias e pneumonias.

\section{CONCLUSÕES}

Com base no levantamento bibliográfico podese aferir que o A. baumannii a cada dia tem ganhado destaque e relevância como agente infecioso no ambiente hospitalar. Verificou uma mudança de seu status de oportunista para micro-organismo multirresistente é importantíssimo. Seu maquinário torna esse micro-organismo persistente e altamente adaptado a hostilidade do meio, além disso o crescente número de mecanismos de resistência certamente o tornam uma espécie temida. Sua significativa participação como agente etiológico em PAVM mostra o quanto é importante estratégias visando métodos profiláticos e preventivo.

\section{REFERÊNCIAS BIBLIOGRÁFICAS}

ANVISA - Agência Nacional de Vigilância Sanitária. (2010). Medidas para identificação, prevenção e controle de infecções relacionadas à assistência à saúde por microrganismos multirresistentes. Brasília.

Chagas TPG. Caracterização de Acinetobacter spp. multirresistentes produtores de carbapenemases, dos tipos OXA e NDM, isolados de diferentes regiões do Brasil. 2015. 133p. Tese (Doutorado em Ciências-Medicina Tropical). Instituto Oswaldo Cruz, Rio de Janeiro, Rio de Janeiro.

Choi CH, Lee JS, Lee YV, Lee JC. Acinetobacter baumannii invades epithelial cells and outer membrane protein A mediates interactions with epithelial cells. BMC Microbiol, v.8, n.1, p.216, 2008.

Chopra S, Galande A. A fluoroquinolone-resistant Acinetobacter baumannii without the quinolone resistance-determining region mutations. Journal of Antimicrobial Chemotherapy, v.66, n.11, p.2668-2670, 2011.

https://doi.org/10.1093/jac/dkr364

Ciello G, Araujo MC. Perfil epidemiológico do Acinetobacter baumannii resistente a carbapenens num hospital do interior mineiro. REFACS (online), v.4, v.3, p.201-207, 2016. https://doi:10.18554/refacs.v4i3.1772

Coelho MJAN. Acinetobacter baumannii uma realidade hospitalar. 2012. 131 p. Dissertação (Mestrado em Gestão e Economia da Saúde). Universidade de Coimbra, Coimbra.

Costa JB; Costa AL; Torres F; Silva AFG; Terra Junior AT. Os principais fatores de risco da pneumonia associada à ventilação mecânica em UTI Adulta. Revista Científica da Faculdade de Educação e Meio Ambiente, v. 7, n. 1, p. 8092, 2016

Davis KA et al. Multidrug-resistant Acinetobacter extremity infections in soldiers. Emerg Infect Dis., v.11, p.12-18, 2005.
Dou Y, Song F, Guo F, Zhou Z, Zhu C, Ziang J; Huan J. Acinetobacter baumannii quorum-sensing signalling molecule induces the expression of drug-resistance genes. Mol Med Rep, v. 15, n. 6, p. 4061-4068, 2017. https://doi:10.3892/mmr.2017.6528

Duarte AFS. Estudos de susceptibilidade e formação de biofilmes de várias estirpes de Acinetobacter baumannii. 2011. 92 p. Dissertação (Mestrado em Bioquímica). Universidade da Beira Interior, Covilhã.

El mekes AZK, Ait said L, Ouafi AT, Barakate M. The clinical and epidemiological risk factors of infections due tomulti-drug resistant bacteria in an adult intensive care unit of University Hospital Center in Marrakesh-Morocco. Journal of Infection and Public Health. v.19, n.1, p.302928, 2019. https://doi.org/10.1016/j.jiph.2019.08.012

Figueiredo DQ, Castro LF, Santos KRN, Teixeira LM, Mondino SSB. Detecção de metalo-beta-lactamases em amostras hospitalares de Pseudomonas aeruginosa e Acinetobacter baumannii. Bras Patol Med Lab, v.45, n.3, p.177$184,2009$.

Gales AC, Jones RN, et al. Global assessment of the antimicrobial activity of polymyxin B against 54,731 clinical isolates of gram-negative bacilli: report from the SENTRY antimicrobial surveillance programme (2001-2004). Clin Microbiol Infect, v.12, n.1, p.315-321, 2006.

Gusatti CS, Ferreira AE, Fuentefria DB, Corção G. Resistência a $\beta$-lactâmicos em Acinetobacter spp. isolados de efluente hospitalar no sul do Brasil. Revista da Sociedade Brasileira de Medicina Tropical v.42, n.2, p.183-187, 2009.

Koneman EW. (2008). Diagnóstico microbiológico: texto e atlas colorido. 6.ed. Rio de Janeiro, Medsi.

Luiz SO. Caracterização da resistência de amostras de Acinetobacter baumannii isoladas no Hospital de Clínicas de Curitiba.2006. 89p. Dissertação (Mestrado em Microbiologia, Parasitologia e Patologia). Universidade Federal do Paraná, Curitiba.

Oliveira AC, Damasceno QS. Superfícies do ambiente hospitalar como possíveis reservatórios de bactérias resistentes: uma revisão. Ver. Esc. Enferm. USP, v.44, n.4, p.1118-23, 2010.

Peleg AY, Adams J, Paterson DL. Tigecycline efflux as a mechanism for nonsusceptibility in Acinetobacter baumannii. Antimicrob. Agents Chemother. v.51, n.1, p.20652069, 2008.

Pereira DCR. Caracterização da resistência de isolados clínicos de Acinetobacter baumannii a antimicrobianos e desinfetante hospitalar. 2013. 104 p. Dissertação (Mestrado em Vigilância Sanitária). Fundação Oswaldo Cruz, Rio de Janeiro.

Perez F, Hujer AM, Hujer KM, Decker BK, RATHER PN, Bonomo RA. Global challenge of multidrug-resistant Acinetobacter baumannii. Antimicrob Agents Chemother, v.5, n.10, p.3471-84, 2007.

Poole K. Efflux-mediated multiresistance in Gram-negative bacteria. Clinical Microbiology and Infection, v.1, n.10, p.2-26, 2004. 
Rello J, Ollendorf DA, Oster G, Vera-Llonch M, Bellm L, Redman R, et la. Epidemiology and outcomes of ventilator-associated pneumonia in a large US database. Chest, v.122, n.6, p.2115-21, 2002.

Rodrigues PMA, Carmo Neto E, Santos LRC, Knibel MF. Pneumonia associada à ventilação mecânica: epidemiologia e impacto na evolução clínica de pacientes em uma unidade de terapia intensiva. J. Bras. Pneumol., v.35, n.11, p.1084-1091, 2009.

https://dx.doi.org/10.1590/S1806-37132009001100005

Sales VM, Oliveira E, Célia R, Gonçalves FR, Melo CC. Análise microbiológica de superfícies inanimadas de uma Unidade de Terapia Intensiva e a segurança do paciente. Revista de Enfermagem Referência, v.4, n.3, p.45-53, 2014.

Silva RNP. A Importância do Acinetobacter baumannii na infecção adquirida nos cuidados de saúde. 2009. 21p. Dissertação (Mestrado Integrado em Medicina) - Universidade do Porto, Porto.

Torres $\mathrm{H}$ et al. Acinetobacter baumanii multirresistente: situación clínica actual y nuevas perspectivas. Revista Espanhola de Quimioterapia, v.23, n.1, p.12-19, 2010.

Uc-Cachón AH et al. High Prevalence of Antimicrobial Resistance Among Gram-Negative Isolated Bacilli in Intensive Care Units at a Tertiary-Care Hospital in Yucatán Mexico. Medicina, v.55, n. 588, p. 1-13, 2019. https://doi:10.3390/medicina5509058

Verma, P; Tiwari, Vishvanath. Targeting Outer Membrane Protein Component AdeC for the Discovery of Efflux Pump Inhibitor against AdeABC Efflux Pump of Multidrug Resistant Acinetobacter baumannii. Cell Biochem Biophys, v.76, n.3, p.391-400, 2018. https://doi:10.1007/s12013-018-0846-5

Zarrilli R et al. Molecular epidemiology of sequential outbreaks of Acinetobacter baumannii in an intensive care unit shows the emergence of carbapenem resistance. J Clin Microbiol., v.42, n.3, p.946-53, 2004.

Zimbler DL. et al. Iron acquisition functions expressed by the human pathogen Acinetobacter baumannii. Biometals, v.22, n.1, p.23-32, 2009. 